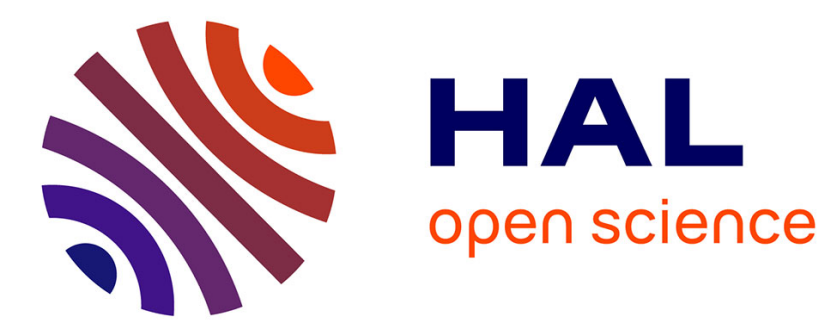

\title{
Efficient Distributed D2D ProSe-Based Service Discovery and Querying in Disaster Situations
}

\author{
Sami Abdellatif, Okba Tibermacine, Walid Bechkit, Abdelmalik Bachir
}

\section{To cite this version:}

Sami Abdellatif, Okba Tibermacine, Walid Bechkit, Abdelmalik Bachir. Efficient Distributed D2D ProSe-Based Service Discovery and Querying in Disaster Situations. AINA 2020 - 34th International Conference on Advanced Information Networking and Applications, Apr 2020, Caserta, Italy. pp.910921, 10.1007/978-3-030-44041-1_79. hal-03094740

\section{HAL Id: hal-03094740 https://hal.science/hal-03094740}

Submitted on 25 Jul 2021

HAL is a multi-disciplinary open access archive for the deposit and dissemination of scientific research documents, whether they are published or not. The documents may come from teaching and research institutions in France or abroad, or from public or private research centers.
L'archive ouverte pluridisciplinaire HAL, est destinée au dépôt et à la diffusion de documents scientifiques de niveau recherche, publiés ou non, émanant des établissements d'enseignement et de recherche français ou étrangers, des laboratoires publics ou privés. 


\title{
Efficient Distributed D2D ProSe-based Service Discovery and Querying in Disaster Situations
}

\author{
Sami Abdellatif, Okba Tibermacine, Walid Bechkit, Abdelmalik Bachir
}

\begin{abstract}
We consider disaster situations where communication infrastructure is partially or totally destroyed. We explore alternatives for enabling fast and efficient service discovery and querying which allow rescuers get access to information produced by available various things such health sensors, wireless cameras, smartwatches and any other relevant devices or sensors helping in the rescue operation. We propose to make use of available users' smartphones for efficiently relaying discovery/querying messages. We make use of LTE Device-to-Device Proximity Services (LTE D2D ProSe) technology to build a distributed D2D broadcast backbone which allows efficient and robust message dissemination avoiding transmission redundancy and resulting in higher energy savings while achieving high coverage. Our solution, Efficient D2D Broadcast (ED2DB) has been implemented on NS-3 by extending the LTE D2D ProSe module provided by NIST. Obtained results show that significant improvements in terms of energy consumption, end-to-end delay, and packet delivery ratio can be obtained by using ED2DB with LTE D2D ProSe over other technologies such as WiFi.
\end{abstract}

Sami Abdellatif

University of Biskra, Algeria, e-mail: sami.abdellatif@univ-biskra.dz

Okba Tibermacine

University of Biskra, Algeria, e-mail: okba.tibermacine@univ-biskra.dz

Walid Bechkit

Univ Lyon, Inria, INSA Lyon, France, e-mail: walid.bechkiteinsa-lyon.fr

Abdelmalik Bachir

University of Biskra, Algeria, e-mail: a.bachireuniv-biskra.dz 


\section{Introduction}

With the recent technological progress, the Internet of Things (IoTs) where a large number of small devices are connected to the global Internet is becoming a reality. Various objects such as wireless cameras, presence sensors, health monitors, smart watches, and many other sensing or actuating devices are already being made discoverable over the Internet and are offering services to many users. These great services things are offering depend on the existence of a reliable communication infrastructure. If something happens to this infrastructure, such as when a disaster hits, these things would become undiscoverable and unreachable remotely. As many of these things, particularly those providing information about the surroundings and health status of victims are crucial for organizing rescue operations, it is very important to promptly establish temporary communication networks as a replacement for the partially or totally destroyed main infrastructure.

There have been many solutions envisaged to achieve this goal, some are based on using Movable and Deployable Resource Units (MDRUs) [1] and others are based on using existing devices to build an Ad-hoc [2] or a Delay Tolerant Network (DTN) [3]. The main drawback of MDRU-based solutions is the deployment of additional units in disaster areas, which could be complex and often leads to delaying the rescue operation. Ad-hoc and DTN based solutions also have shortcomings as they are typically built over short range wireless technologies such as WiFi, which may lead to fragmented sub networks thereby increasing transmission delays at best and failing to achieve coverage at worst.

Recently, Third Generation Partnership Project (3GPP) in its Releases 12 and 13 proposed a technology based on LTE device-to-device (D2D) communication called Proximity Service (ProSe) with communication ranges significantly greater than the usual technologies adopted in the aforementioned solutions [4]. ProSe communication means a communication between two or more User Equipment (UEs) that are in proximity of each other can take effect directly without passing through the LTE core network [5]. Due to the promising qualities of ProSe (mainly long range), many research efforts are considering it for building more efficient replacement networks. For instance, in [6], the authors proposed to collect health related information from wearable devices and on-body sensors using victim's smartphone. The collected information is then broadcast to nearby smartphones using ProSe to reach more people. However, only a one-hop broadcast is considered, and the presence of Internet or a partial-coverage communication infrastructure is mandatory to enable D2D direct communication, which might not be suitable in case of large-scale disaster.

In [7], the authors addressed the problem of multicasting over LTE D2D ProSe to perform file distribution to group members. The authors, proposed a selection of relay nodes performed by a mobile eNodeB installed on a vehicle, where the selected nodes re-transmit data to all other remaining nodes. The selection process reduces the number of broadcasting nodes, which resulted in better performance than broadcasting directly to reach all nodes (i.e. flooding over ProSe). Although this solution proposes a multihop broadcast over ProSe, its use is only limited in partialcoverage situations. In addition, this solution is centralized as it mainly depends on 
the mobile eNodeB to perform node discovery and selection, as well as initiate file distribution over a tree based topology.

In our proposal, we extend the one-hop LTE D2D ProSe broadcast to support multihop communication in an out-of-coverage scenarios. We propose Efficient D2D Broadcast (ED2DB) which provides significant performance improvement over flooding which suffers from high overhead and energy consumption due to redundant transmissions. We design a distributed solution where every UE performs discovery, relay selection and can initiate communication. In addition, we take into consideration the difference between UEs and other IoT devices and that the discovery and interconnection of these IoT devices affect the construction of the replacement network structure, i.e. the number of IoT devices and their distribution over UEs is an important factor to consider during the selection of relays. Thus, the replacement network becomes heterogeneous in terms of wireless technologies, protocols, standards and modes of operations used between UEs and IoT devices.

In regards to the limitation and shortcoming of the previously mentioned solutions, our solution proposes the following:

- a distributed multihop LTE D2D ProSe broadcasting structure for efficient discovery/querying message dissemination.

- addressing the heterogeneity of nodes in the networks UEs and IoT devices.

- Quantifying the benefits of using a long range LTE D2D ProSe on message delivery ratios, energy consumption, and transmission delay compared to the traditional and widely used WiFi-based solutions.

The remainder of this paper is organized as follows: Section 2 and 3 presents the network model and formulate the problem statement of this work. Section 4 describes the provisional network construction in a disaster situation. Section 5 presents the implementations and experimentation details. Section 6 discusses the results obtained by the proposed solution using WiFi and LTE ProSe. Section 7 concludes the paper and provides some future directions.

\section{Network Model and assumptions}

We use the following terminology where we distinguish between Device, Station, Relay and Node. We call a Device any small thing capable of performing sensing or actuating and equipped with limited storage, processing and communication resources. We call Station any higher capacity communicating object, typically a smart phone equipped with LTE technology and capable of performing D2D ProSe. A Node can be either a Device or a Station. A Relay is a Station involved in data forwarding from a Node to other Nodes.

We model the network in a disaster affected area with no cellular coverage as a graph $G(S, D, E)$ where $S$ is the set of Stations which are typically smartphones held by rescuers, volunteers, or victims. We assume that rescuers, volunteers and victims have LTE D2D ProSe enabled smartphones and also already installed our 
disaster relief application on their smart devices which allows them to (i) take part in establishing replacement network and communicate with other peers directly via LTE D2D ProSe, and (ii) discover and collect data from surrounding IoT devices via other interfaces (i.e., Bluetooth, BLE, ZigBee, WiFi etc), as well as (iii) exchange of localization information, help messages and other data in text, image, audio and video formats. $D$ is the set of IoT Devices (e.g. vital signs monitoring devices, smartwatches, wearable, etc.) $E$ is the set of edges where $E_{i j}$ denotes a direct wireless link from $i$ to $j$, such that $i$ and $j$ belong to $S \cup D$.

We assume that rescuers and volunteers are mobile and spread all across the affected area and victims are stationary waiting to be rescued. We assume that all stations are equipped with a USIM which has all the pre-configuration and authorisation necessary for LTE D2D ProSe Communication specified in [5]

\section{Problem Formulation}

The construction of a broadcast-centric network structure is primordial to cope with disaster situation requirements as it makes remove service discovery and querying of IoT devices possible again even in the absence of the main infrastructure. A group communication between rescue team should be also facilitated by exploiting the infrastructure. Moreover, it allow rescuers (or any other entity that uses the network) to query a group of devices or stations simultaneously, in order to collect data from the environment.

Obviously, using a flood routing, which involves all stations during communication, leads to generate unnecessary traffic with high level of contention at the MAC layer that causes performance degradation.

Wasting energy is a major drawback of this type of solutions. For instance a number of stations constantly querying a device or re-transmit messages will result in faster battery depletion of both devices and all querying stations. Thus, the main goal is to minimize packet transmissions, as well as maximize battery life and packet delivery ratio, through the consideration of an efficient D2D broadcast. This mechanism operates on a network of a minimal number of relays that ensures a full coverage and connectivity between stations and devices. The selection of these relays is not a trivial task but should be done in a way that ensures the aforementioned requirements.

Problem statement: Given the network graph $G(S, D, E)$ previously defined, let the ProSe cost, denoted $P\left\{n_{s}, n_{d}\right\}$ be the cost incurred by any packet transmission from source node $n_{s}$ to destination node $n_{d}$ over multi-hop broadcast where $n_{s}$ and $n_{d}$ belongs to $S \cup D$. To minimise the ProSe Cost, the replacement network should be constructed tacking in account the following criteria : (i) use a minimum number of relay nodes that can broadcast any packet from source $n_{S}$ to destination $n_{d}$, and (ii) select the optimal set $M \in S$ of relay nodes that ensures network connectivity, (iii) and use all smartphone candidates that cover a maximum number of IoT devices. The problem of minimizing the number of ProSe broadcast taken is NP-complete. 
We prove this by showing that the above problem is similar to the minimum Connected Dominating Set (CDS), which is already known as NP-complete [8]. However, the problem is more complex because it considers two type of nodes (Station and IoT Devices). Next, we discuss in detail the proposed heuristic to tackle the aforementioned problem.

\section{Proposed Solution}

In this section, we discuss the selection of relay stations, in such a way that the overall ProSe cost is minimized. For the sake of simplicity, we consider that each device has only one interface and each smartphone has many. The communication between smartphones occurs over LTE D2D ProSe interface.

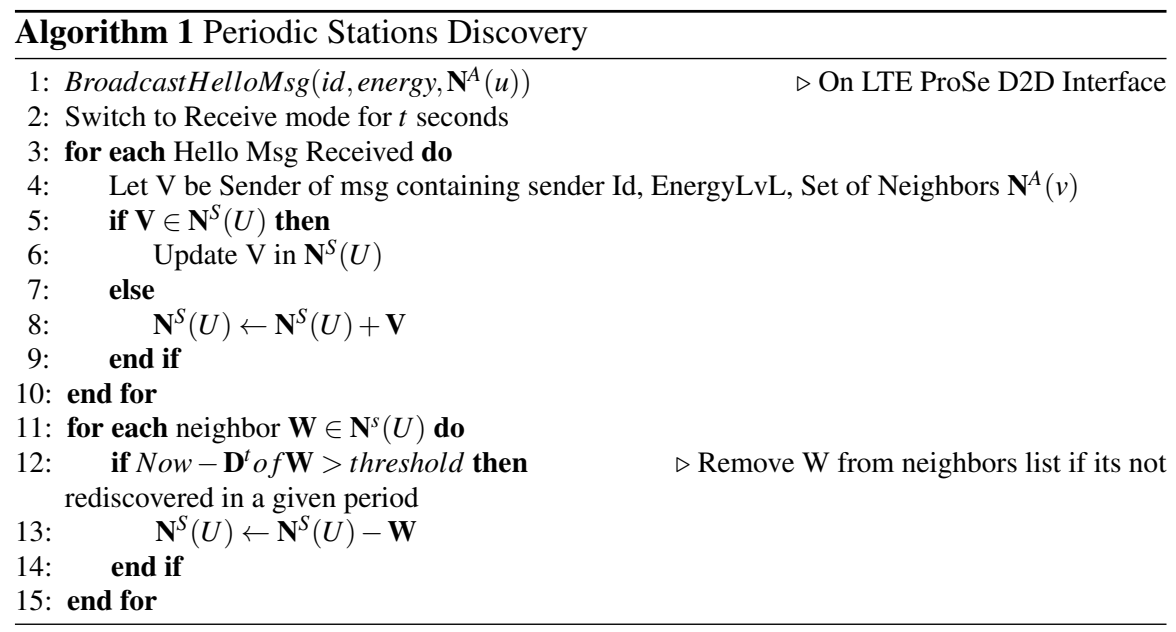

\subsection{Discovery Phase}

In the first phase, each smartphone (station) starts to discover its surrounding devices and neighboring smartphones.

\subsubsection{Periodic Station Discovery}

In this phase every station sends hello messages to neighboring stations over periodic broadcast transmissions. The discovery message contains information related 
to energy level, neighbours list and the degree of connectivity which are necessary parameters for the next phases. Although 3GPP specifies a direct device to device (D2D) discovery protocol that allows for a station to discovery its neighbors, the ProSe discovery mechanism works only in coverage and in partial-coverage situations and require the presence of LTE core network. in addition, the ProSe specification imposes a certain message structure and a limited size. Thus, including the aforementioned discovery information in ProSe discovery messages is unpractical. To enable station discovery, we propose another mechanism that is based on ProSe broadcast communication, where stations periodically broadcast hello messages containing the aforementioned information. Algorithm 1 presents the discovery phase of surrounding stations over LTE D2D ProSe direct communication.

\subsubsection{Periodic Device Discovery}

To ensure a maximum coverage of IoT devices, stations periodically discover their surrounding devices. The number of devices discovered by a station is an important factor during the selection of relay stations. In fact, smartphones support a large set of wireless interfaces (WiFi, LTE, Bluetooth, ZigBee, ANT+, NFC, QR, etc.) [9]. Moreover, smartphones are capable of communicating with different types of smart objects such as devices used in smart-homes, healthcare systems, Wireless cameras or other ProSe enabled smartphones. Stations discover devices in two modes; (i) either listening to hello messages from devices that support push mode (e.g. Google Eddystone, iBeacon, AliveCor [10]), or (ii) broadcasting a "Who is there" message to discover devices operating in pull mode (e.g. wireless cameras). A station has to switch constantly between interfaces in order to send discovery messages. A station, stores discovered devices, services, communication mode and interfaces in a table. Algorithm 2 presents devices discovery phase.

\subsection{Distributed Relay Selection}

Using discovery results obtained from Phases I and II (station and device tables), we construct a network backbone. Stations that belong to the backbone are referred to as Broadcasting nodes and are the only nodes responsible for data forwarding. After the previous discovery phases, each node is aware of its neighboring station and devices. If a station detects that two of his neighbors are not connected directly, then the station becomes a relay station. If two stations $S_{1}, S_{2}$ with set of neighbors $\mathbf{N}^{A}\left(S_{1}\right)$, $\mathbf{N}^{A}\left(S_{2}\right)$ respectively, and $\mathbf{N}^{A}\left(S_{1}\right) \subset \quad \mathbf{N}^{A}\left(S_{2}\right)$ then $S_{1}$ is considered a simple station, otherwise $S_{1}$ becomes a relay station. In case where $\mathbf{N}^{A}\left(S_{1}\right)$ equals $\mathbf{N}^{A}\left(S_{2}\right)$ then we favor the station with the higher residual energy. After this, each selected station is assigned a set of devices that it manages. A device can connect to more than one station. This phase is repeated periodically over a fixed time interval, network changes can also be considered as a triggering factor, by monitoring the changes 


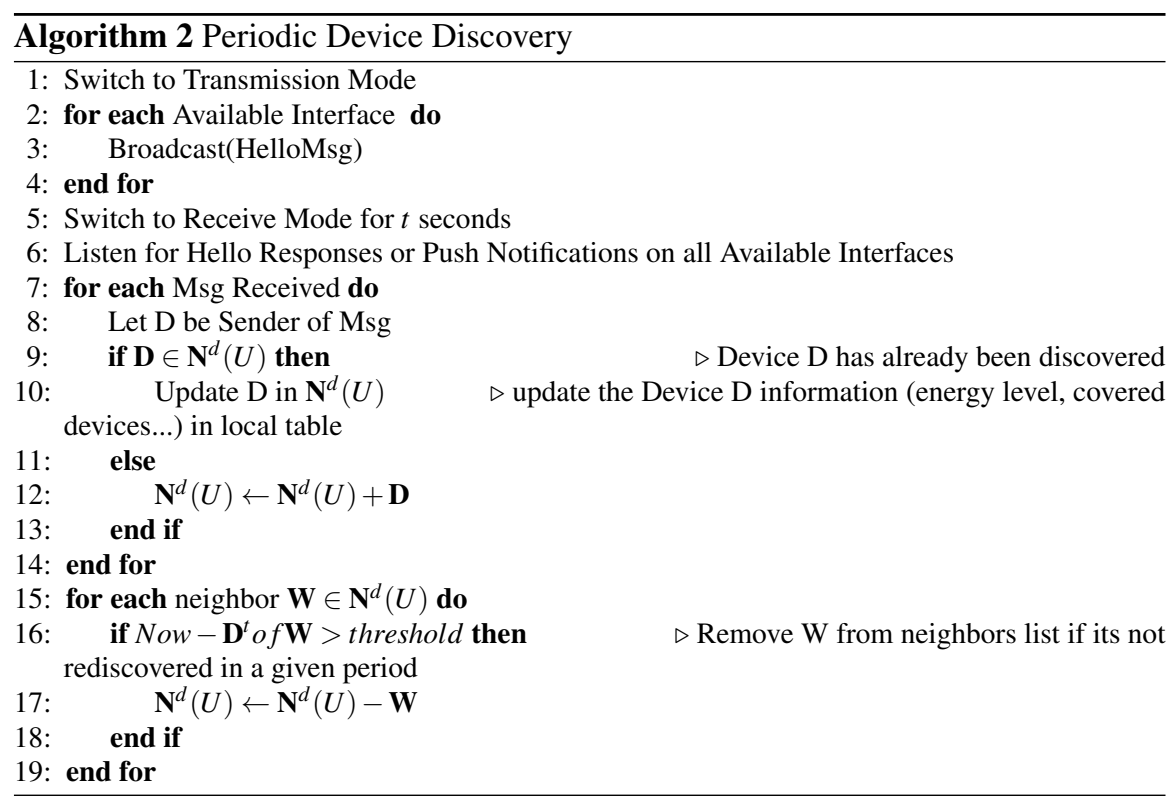

ratio of every local table, and if it exceeds a selected threshold value then a selection process will be triggered. Algorithm 3 shows the periodic selection process .

\subsection{Efficient D2D Broadcast}

To be able to route information to their destination, we foresee two modes of operation push and pull.

In push mode, devices would push their data to a nearby relay station, this station will broadcast the aggregated data to all its neighboring relays and so on until all rescuers are informed so they can take the proper decision in the right time.

In pull mode, the entity interested in the service or data initiates the process by sending a request or a query for a given service. For example if a Rescuer $\mathrm{R}$ is interested in the health status of a victim under debris, then he will start by sending a request to the heart monitor attached to the victim (smart-watch), this request must go through the station covering the needed device. After receiving such request the station then queries the device for its current data and then sends the result to the rescuer. Pull mode may consume less resources (energy, transmission, time) than the push mode. However, the push mode gives rescuers a global knowledge of the entire area in real time. Algorithms 4 shows the procedure of routing Requests and Responses messages. 


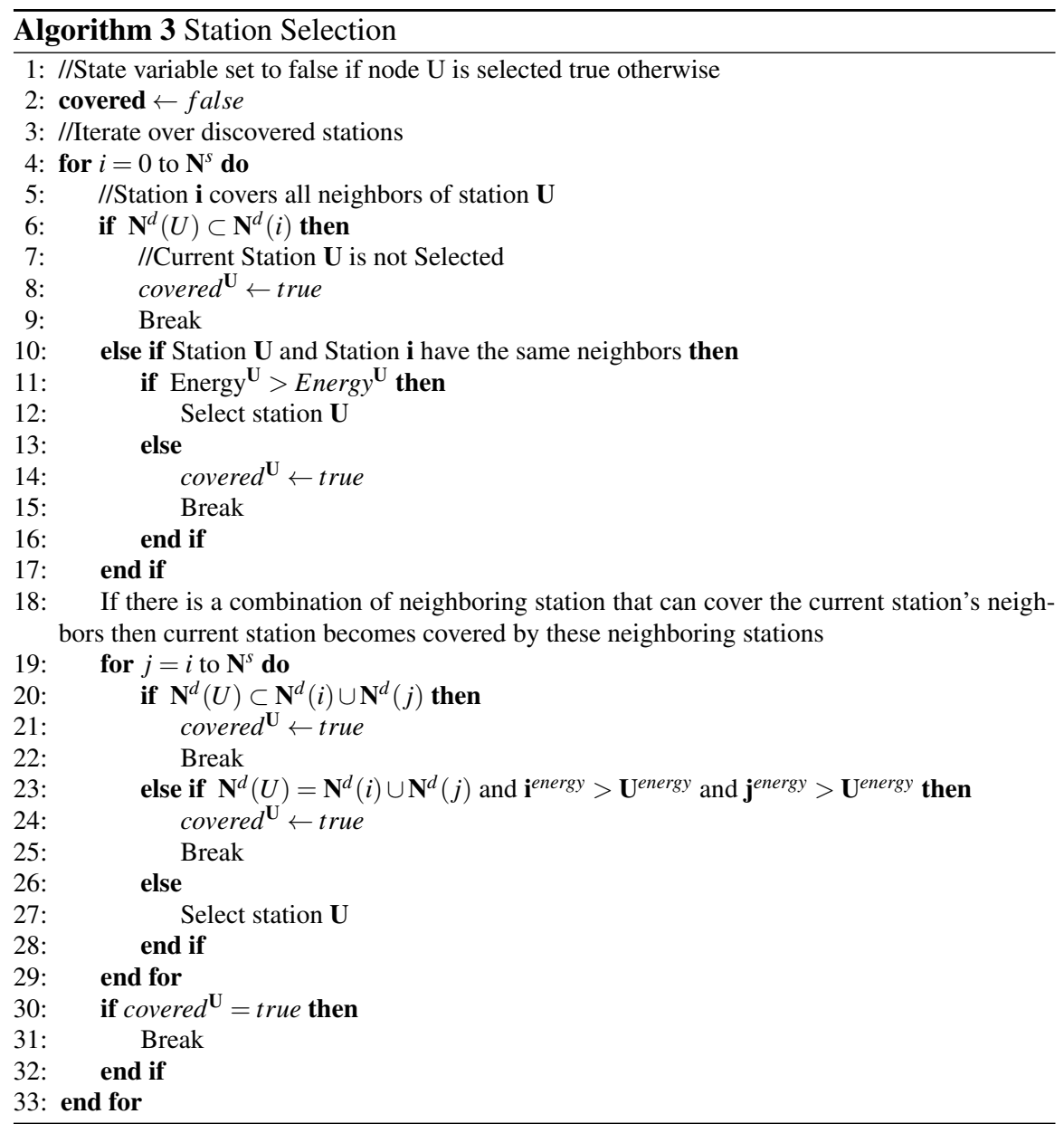

\section{Evaluation}

In this section, we present an evaluation of the proposed solution through simulation in the discrete event network simulator ns-3 [11]. We implemented our scenarios on top of the LTE ProSe module provided by NIST [12]. Nodes are deployed in an area of $500 \mathrm{~m} * 500 \mathrm{~m}$ randomly following a uniform distribution and divided into two groups, stations and devices.Stations are mobile nodes following the random walk model, with a variety of wireless interfaces, namely LTE, 802.11 (Wi-Fi) and 802.15.4. Each station is equipped with an energy source of $10000 \mathrm{~J}$. The rest of nodes (IoT devices) are stationary and randomly deployed across the simulation area. Devices only support 6LoWPAN over 802.15.4 and operate on an energy source of 100J. We use the same Cost-231-Hata [13] propagation model across all our simulation scenarios. 


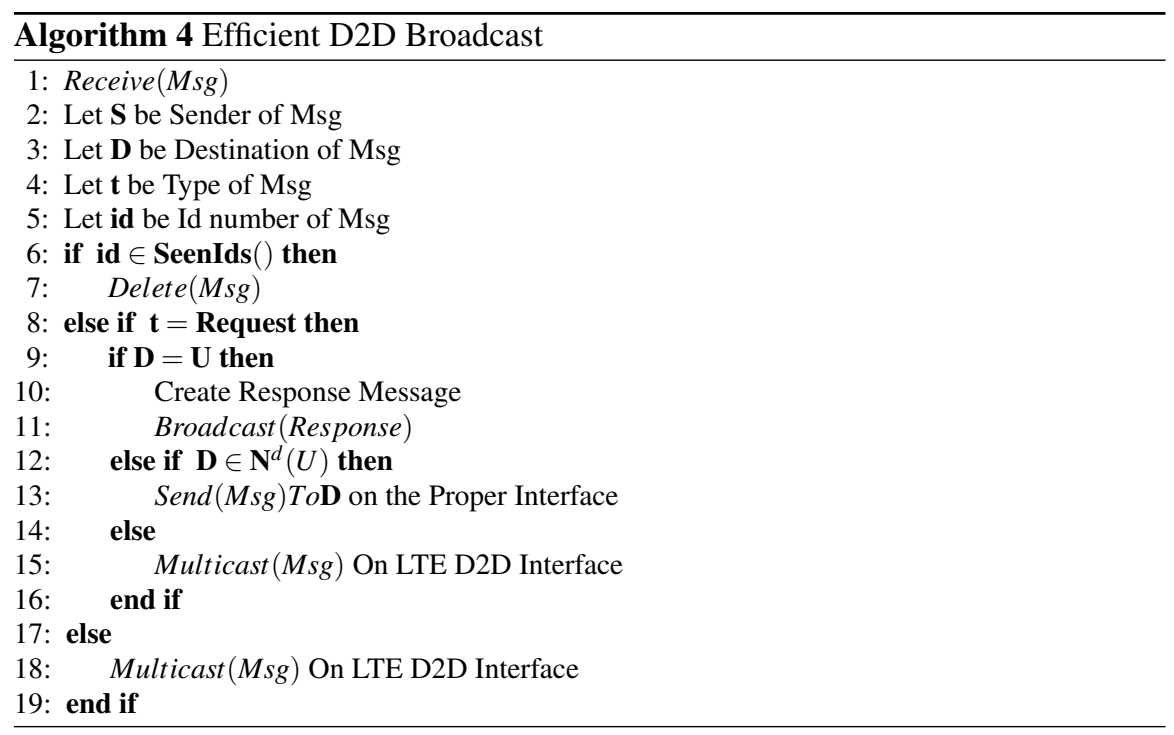

In our simulation only stations perform the selection process. Data traffic consists of request/response packets and follows a Poisson process with 10 seconds time interval. For simulation purposes, we examine different network sizes.

\subsection{Scenarios}

Inn order to evaluate the performance gains provided by ED2DB over ProSe, in terms of low consumption of battery power, high delivery ratio and low end-to-end delay, our algorithm has been compared with three other algorithms namely :

(i) Epidemic routing over WiFi [14]: where all station broadcast packets when received until it reaches destination. This protocol is highly used in disaster relief solution due to its robustness and high delivery packet ratio.

(ii) Epidemic routing over LTE D2D ProSe: We adopted the existing epidemic algorithm and implemented it over LTE D2D ProSe to evaluate; (i) the performance of the long range LTE D2D ProSe in contrast to traditional WiFi. (ii) the cost of uncontrolled broadcast transmissions over LTE D2D ProSe.

(iii) ED2DB over WiFi: we proposed to build the network structure over WiFi to route packets.

Our simulation is repeated 100 times, and the results were analyzed with a 95\% confidence interval. Our goal is to compare our work with the three mentioned algorithms in a disaster situation. 


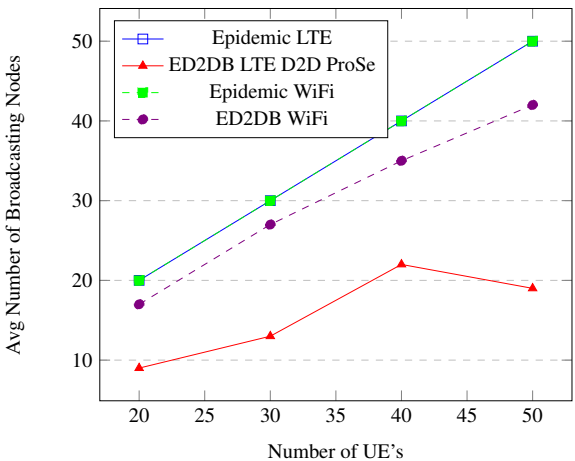

Fig. 1 Number of Broadcasting Nodes in function of Network Size

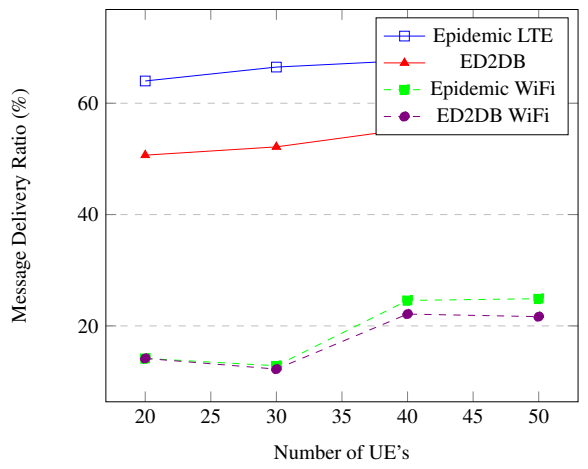

Fig. 2 Message Delivery Ratio in function of Network Size

\section{Results and Analysis}

We evaluated the performance of the aforementioned scenarios and compared them regarding different metrics.

\subsection{Effect of Number of Broadcasting Nodes}

Figure 1 shows that, in case of our algorithm only a small portion of the existing stations are selected by our algorithm as broadcasting nodes, leaving more than half the nodes in idle state. Whereas, in case of ED2DB on WiFi and the two epidemic approaches, most stations are used to forward packet from end-to-end. Having less nodes involved in packet forwarding can lead to a decrease in energy consumption and less network overhead meaning a better network overall performance.

\subsection{Reachability}

In case of a disaster it is very important that a victim's help message can reach a rescuer as well as hello packets generated by a rescuer can reach the victims. To this end we set this metric as the ratio of messages successfully delivered to the total sent. As shown in Figure 2, the proposed algorithm outperforms ED2DB on WiFi in terms of request resolution and scales well with networks density since more stations translate to better coverage and connectivity. While it guarantees the same level of reachability as epidemic routing with more than half the nodes inactive. Figure 3 explains the previous results, as it shows that both the proposed solution 


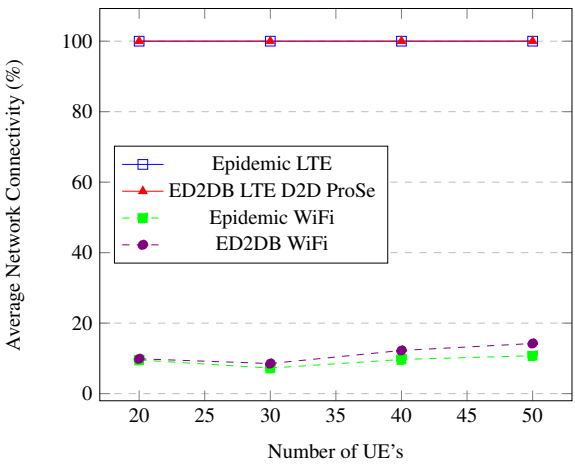

Fig. 3 Average Network Coverage in function of Network Size

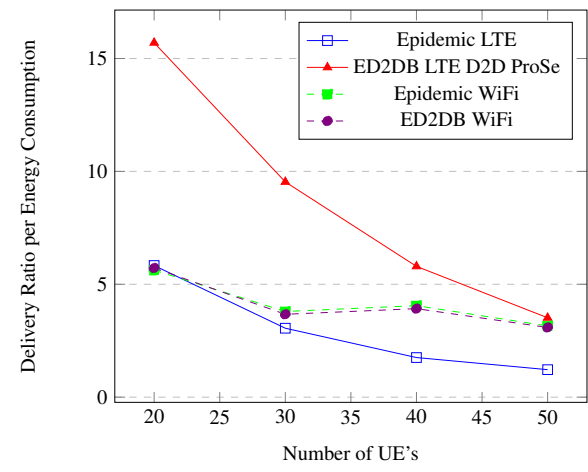

Fig. 5 Delivery Ratio per Energy Consumption in function of Network Size

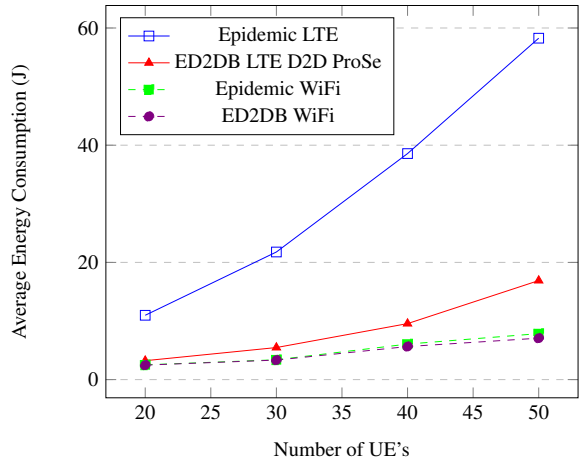

Fig. 4 Average Energy Consumption in function of Network Size

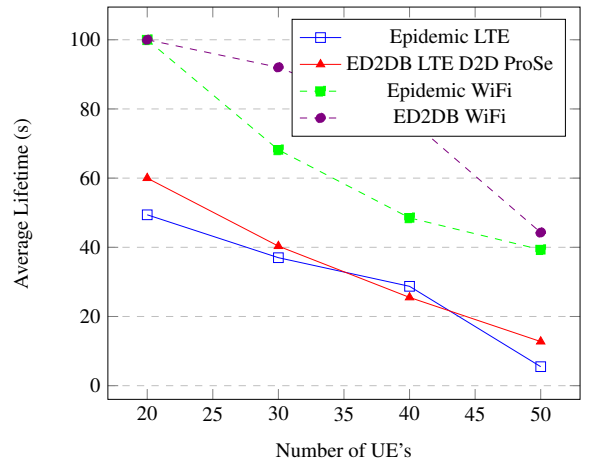

Fig. 6 Average Lifetime in function of Network Size

and epidemic routing generate a better connected network, meaning that no matter where the victim is there is a path between him and at least a rescuer.

\subsection{Stations Energy Consumption}

A Primary goal of ED2DB is energy consumption. As shown in Figure 4 ED2DB outperforms the epidemic routing, this shows that ProSe message broadcasting must be controlled other wise it results in a degradation in performance. However, we notice that ED2DB on WiFi consumes less that ED2DB on LTE D2D ProSe. Nonetheless, Figure 5 shows that when we look at energy consumption per delivery ratio it's clear that the ED2DB on ProSe is far more superior meaning that it's more balanced than the others in terms of Energy/Delivery ratio. And to better assess the energy 


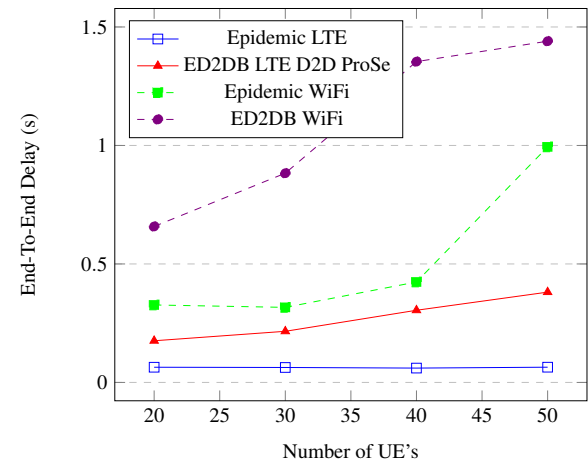

Fig. 7 End-to-End Transmission Delay in function of Network Size

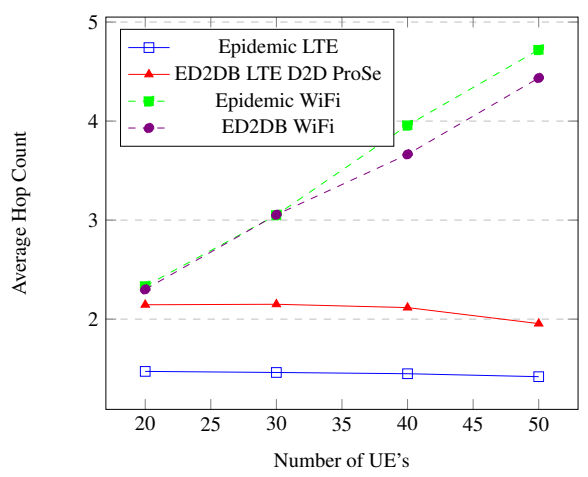

Fig. 8 Average Hop Count in function of Network Size

consumption we measured the life time of network, where we set the residual energy of smartphones to $20 \mathrm{j}$ and run the simulation until $20 \%$ of nodes are dead. Figure 6 shows that ED2DB on WiFi outperforms our solution where it allows smartphones to live longer, however during their life span stations remain ineffective.

\subsection{End-To-End Transmission Delay}

Here, we compare the average time required for delivering a packet to its destination. Figure7 7 shows end to end transmission delay with respect to the network size. We observe that WiFi-based solutions present higher delay compared to ProSe-based solutions. This is because WiFi uses a higher hop count to deliver a packet to its destination as showed in Figure 8 . This small hop count could be explained by the long range of ProSe interface, where in case of WiFi more hops are required to deliver packets successfully.

\section{Conclusion}

We have proposed an efficient D2D broadcast on top of the ProSe direct communication for out of coverage situatins. ED2DB enables rescue teams to communicate and discover their surroundings, as well as allowing victims to reach rescuers via help messages and offer rich information about their state. In addition, the network structure allows for connecting and giving access to IoT devices. The proposed solution insures a maximum connectivity and coverage by strategically selecting a number of stations with higher energy levels and connectivity degree. We have shown that contrary to simply using ProSe broadcasting, selecting the right broadcasting nodes 
yield significant performance gains, especially in preserving network lifetime. In future work, we will focus on balancing the load on different stations to avoid exhausting the well connected ones. In addition, we will address the dynamic and hostile nature of disaster environment by suggesting a fault tolerant solution that relies on deploying complementary backups in case a relay is off.

\section{References}

1. T. Sakano, S. Kotabe, T. Komukai, T. Kumagai, Y. Shimizu, A. Takahara, T. Ngo, Z. M. Fadlullah, H. Nishiyama, and N. Kato, "Bringing movable and deployable networks to disaster areas: Development and field test of MDRU," IEEE Network, vol. 30, no. 1, pp. 86-91, 2016.

2. D. Reina, M. Askalani, S. Toral, F. Barrero, E. Asimakopoulou, and N. Bessis, "A survey on multihop ad hoc networks for disaster response scenarios," International Journal of Distributed Sensor Networks, vol. 11, no. 10, p. 647037, 2015.

3. S. Saha, A. Sheldekar, A. Mukherjee, S. Nandi, et al., "Post disaster management using delay tolerant network," in Recent Trends in Wireless and Mobile Networks, pp. 170-184, Springer, 2011.

4. Qualcomm, "Lte direct trial whitepaper." https ://www. qualcomm. com/documents / lte-direct-trial-white-paper 2015. Accessed: 2019;12;3.

5. Universal mobile telecommunications system (UMTS), proximity-based services (ProSe); Stage 2, Apr 2016. Rev. 12.0.

6. P. Bellavista, J. De Benedetto, C. R. De Rolt, L. Foschini, and R. Montanari, "Lte proximity discovery for supporting participatory mobile health communities," in Communications (ICC), 2017 IEEE International Conference on, pp. 1-6, IEEE, 2017.

7. S. I. Sou, M. R. Li, S. H. Wang, and M. H. Tsai, "File distribution via proximity group communications in LTE-advanced public safety networks," Computer Networks, vol. 149, pp. 93-101, 2019.

8. M. Chlebík and J. Chlebíková, "Approximation hardness of dominating set problems in bounded degree graphs," Information and Computation, vol. 206, no. 11, pp. 1264-1275, 2008.

9. G. Aloi, G. Caliciuri, G. Fortino, R. Gravina, P. Pace, W. Russo, and C. Savaglio, "Enabling iot interoperability through opportunistic smartphone-based mobile gateways," Journal of Network and Computer Applications, vol. 81, pp. 74-84, 2017.

10. AliveCor, "Alivecor kardiaband." https://store.alivecor.com/products/ kardiaband april 2019.

11. G. F. Riley and T. R. Henderson, "The ns-3 network simulator," in Modeling and tools for network simulation, pp. 15-34, Springer, 2010.

12. R. Rouil, F. J. Cintrón, A. Ben Mosbah, and S. Gamboa, "Implementation and validation of an lte d2d model for ns-3," in Proceedings of the Workshop on ns-3, pp. 55-62, ACM, 2017.

13. G. F. Pedersen, "Cost 231-digital mobile radio towards future generation systems," in Cost 231-Digital Mobile Radio Towards Future Generation Systems, pp. 92-96, EU, 1999.

14. A. Vahdat, D. Becker, et al., "Epidemic routing for partially connected ad hoc networks," 2000 . 